\title{
Revista Andina de Educación
}

http://revistas.uasb.edu.ec/index.php/ree

\section{Rutas de aprendizaje en la construcción de narrativas transmedia apli- cadas a aulas virtuales \\ Learning paths in the construction of transmedia narratives applied to virtual classrooms}

\author{
Gilberto Natividad Aranguren Peraza ${ }^{a}$ \\ ${ }^{a}$ Universidad Nacional Experimental Simón Rodríguez. Santa Fe Norte, Av. José María Vargas, Torre del Colegio de \\ Médicos, piso 7. 1080 Caracas, Venezuela.
}

\section{INFORMACIÓN DEL ARTÍCULO}

Historial del artículo:

Recibido el 19 de octubre de 2020

Aceptado el 14 de febrero de 2021

Publicado el 15 de febrero de 2021

Palabras clave:

Rutas de Aprendizaje

Aula Virtual

Narrativas Transmedia

ARTICLE INFO

Article history:

Received October 19, 2020

Accepted February 14, 2021

Published February 15, 2021

keywords:

Learning paths

Virtual classroom

Transmedia narratives

\begin{abstract}
RESUMEN
El objetivo del ensayo es analizar el alcance de las rutas de aprendizaje en la construcción de narrativas transmedia aplicadas a aulas virtuales. Las narrativas transmedia son sistemas construidos con la participación colaborativa y la integración de estrategias, medios, recursos y plataformas para orientar los contenidos. Se plantea un análisis documental mediante una reflexión y argumentación teórica. Una conclusión es que las rutas de aprendizaje son recursos que facilitan la conexión de diferentes medios que dirigen la enseñanza a través de narrativas que requieren de estrategias colaborativas para la reorganización de las ideas, del aprendizaje y la interacción.
\end{abstract}

ABSTRACT

The essay's objective is to analyze the scope of the learning paths in the construction of transmedia narratives applied to virtual classrooms. Transmedia narratives are systems built with collaborative participation and the integration of strategies, media, resources, and platforms to guide content. Documentary analysis is proposed through reflection and theoretical argumentation. One conclusion is that learning routes facilitate the connection of different media that direct teaching through narratives that require collaborative strategies to reorganize ideas, learning, and interaction.

(C) 2021 Aranguren Peraza CC BY-NC 4.0

\section{Introducción}

La complejidad está basada en tejidos dados entre variables que componen la vida cotidiana, donde se mezclan aspectos como la economía, la política y la cultura con construcciones mentales referidas a lo afectivo y psicológico. La noción de complejidad supone un conglomerado de redes de pensamientos interconectados construidos en la medida que la persona interactúa y aprende, considerando, para ello, la relación entre la unidad con la multiplicidad (Morín, 1999). Esto conduce a pensar en nuevos modos de concepción de los procesos educativos (Ortiz, 2018), aún más, cuando se experimentan situaciones extremas como el confinamiento y distanciamiento físico como protección ante las condiciones dadas por la pandemia del COVID-19 a nivel mundial (OPS, 2020).

La complejidad ha facilitado la educación desde una perspectiva virtual, revelándose en ello una serie de problemas de índole político-cultural tales como la llamada

\footnotetext{
*Autor principal: Gilberto Natividad Aranguren Peraza. Correo electróni-
} co: gilberap@gmail.com falacia del diálogo virtual, el dominio de las minorías, la información como sustituto de la formación, el concepto de aprendizaje activo como excusa para una asistencia limitada, el debilitamiento de la comunidad educativa, entre otros (Ralón, Vieta, \& Vásquez, 2004).

El acceso a la educación virtual en muchos casos es difícil, no solo por el hecho que a muchas personas se le hace compleja la relación con el mundo virtual, dado que la enseñanza presencial representa el modo en que, por muchos siglos, la persona ha comprendido los asuntos referidos a su aprendizaje, sino porque estas transformaciones vienen dándose mediante procesos adaptativos que suponen cambios sustanciales a nivel de los estados mentales. Entendiendo que los estados mentales se determinan en la mente y está no funciona sin el cuerpo, porque en la mente se integran los pensamientos, emociones, sentimientos y espiritualidad, afectándose los sistemas cognoscitivos, los estados de ánimo, y las actitudes frente a las situaciones problemáticas de la vida (Aranguren, 2020).

Es paradójico hablar del aprendizaje virtual en la sociedad del conocimiento, cuando más de la mitad de la población 
mundial no tiene acceso a internet, ya sea por las dificultades en el uso de la tecnología o porque las censuras limitan los modos en que se debe distribuir la información, sumándose a ello las limitaciones económicas para la adquisición de equipos, infraestructura y formación (Olarte, 2017).

En este contexto, han surgido Sistemas para la Gestión de Aprendizaje (Learning Management Systems), software empleado para la administración, distribución y control de actividades de formación, aulas virtuales, llamadas también Entornos Virtuales de Enseñanza o de Aprendizaje (EVA). Entre las plataformas más conocidas se encuentran Moodle, Web Course Tool (WebCT), Learningspace y Blackboard, siendo importante destacar el Basic Support for Cooperative Work (BSCW), plataforma orientada al desarrollo de procesos colaborativos en la Red (García-Valcárcel, Muñoz-Repiso, \& Hernández, 2013). En tal sentido, Fernández-Pampillón (2009) define los EVA como plataformas e-learning capaces de crear realidades tecnológicas, diseñadas para ofrecer un espacio para la enseñanza y el aprendizaje, a diferencia de los contextos o ambientes de enseñanza tradicional que sostienen su acción en las clases presenciales (Gil, \& Mataveli, 2018). Los EVA son ecosistemas pedagógicos virtuales capaces de mediar los procesos de enseñanza y aprendizaje mediante un sistema comunicacional y de interacción que permite el desarrollo integral de estrategias, actividades, procesos de evaluación y accesibilidad mediante una serie de formatos, ya sean estructurados como las aulas virtuales, o no estructurados como las redes sociales (Domínguez, 2016).

Las aulas virtuales son entornos tecnológicos (Colás, 2006) o ambientes de aprendizaje y enseñanza (Monroy, Hernández, \& Jiménez, 2018), sostenidas en plataformas virtuales que integran recursos, herramientas, actividades, tareas y estrategias de índole educativo, formativo y de capacitación (FAO, 2014), convirtiéndose, hoy día, en una necesidad por facilitar el acceso a recursos sin límites espacio-temporales y ofreciendo la posibilidad de interacción y comunicación mediante aportes colaborativos.

En contraste con la educación presencial, el uso de las aulas virtuales tienden a ser de bajo costo en consideración con "los costos asociados a las instalaciones para las salas de clases, el tiempo de los instructores, y el tiempo de viaje de los alumnos y de ausencia de su trabajo para asistir a clases" (FAO, 2014). De manera, que los procesos de formación y capacitación pueden llegar a un público más amplio del que se supone está permitido en una clase presencial, siendo de provecho para aquellas personas con poco tiempo y recursos para el traslado, y también para aquellas con compromisos que impiden su asistencia con regularidad a las clases presenciales; además, lo asincrónico facilita la conexión en horarios y fechas diferentes.

Las aulas virtuales son una opción para la enseñanza dado que en ellas puede darse el aprendizaje de forma distribuida y colaborativa, respondiendo a lo esperado en las competencias y en los objetivos planteados; el desarrollo de la enseñanza a través de narrativas transmedia construidas mediante rutas de aprendizaje, facilitan la organización y orientación de los contenidos, de los métodos y estrategias para la enseñanza virtual (FAO, 2014) y de las actividades para la adquisición y procesamiento de la información, haciendo de estas plataformas alternativas eficaces y de gran alcance en los procesos de formación y capacitación (García, 2018) y contribuyendo con el acercamiento entre el estudiantado y el profesorado.

Por ello, requieren ser gestionadas mediante contenidos, actividades, estrategias y procesos de seguimiento y evaluación que respondan a los objetivos y competencias planteadas, mediante el uso de recursos y herramientas tecnológicas que consoliden la enseñanza, por lo que es necesario comprender que las tecnologías son canales mediadores del aprendizaje y del conocimiento; por consiguiente, esta noción coloca al docente a pensar en la clase bajo una lógica de las narrativas.

En el contexto de la enseñanza, pensar en una clase, inequívocamente nos lleva a pensar en algún tipo de relato. En las clases se enseña claramente en forma narrativa. Los relatos se usan en clase para convencer, entretener, intrigar, explicar, impresionar, teorizar y crear vínculos. Entendemos la narración como una voz dentro de un contexto cultural

(Santoveña-Casal, 2019, p. 26)

Estas narrativas deben conducir, entre otras cosas, la interacción, la comprensión y el desarrollo de la cognición, y para la consecución de esa meta se requieren de relatos debidamente organizados. El enfoque de las rutas de aprendizaje presentados por Hernández-Bieliukas y Aranguren (2016), se sostiene en los principios que orientan la adquisición de las habilidades cognitivas (Chadwick, 1991; Gilar, 2003; Castejón, \& Gilar, 2005), se basa en estrategias que facilitan, no solo la organización del contenido, sino incluso la comprensión, el desarrollo de habilidades y el logro de las competencias; por ello, se plantea un análisis acerca del alcance de las rutas de aprendizaje en la construcción de narrativas transmedia aplicadas al desarrollo de los contenidos en las aulas virtuales, entendidas éstas como EVA.

\section{Las aulas virtuales, la interactividad y el aprendizaje}

Para los años sesenta del siglo XX, los sistemas educativos y con ellos la escuela se ven acompañados de los que para entonces representaban los modernos medios audiovisuales; la tecnología educativa iniciaba un proceso acelerado de crecimiento, evidenciándose transformaciones en las TIC (Bautista, 2004), en los sistemas reproductores de la información y datos, en las interacciones y estructuras simbólicas de representación que sostenían a los sonidos e imágenes y en las actitudes, percepciones y razonamiento del estudiantado y profesorado (Moreno, 2018).

Posteriormente, con el desarrollo de internet, los procesos de Tecnología Educativa aunados al auge de las TIC facilitaron la consolidación de los EVA, fortaleciendo los procesos de adquisición, procesamiento y transferencia de la información y profundizando la formación a distancia (Malbrán, 2012; Tapia, 2016; Reyero, 2019).

El prestigio de esta modalidad, en algún momento cuestionado, ha crecido en los últimos años. Se han creado universidades que trabajan solo en esta modalidad. Las universidades tradicionales, que por años han realizado clases solo presenciales, han empezado a incluirlas como apoyo a la clase presencial, como complemento o han decidido incorporar un área íntegramente virtual

(Silva, 2011, p. 20)

Estas tecnologías favorecen la interacción y comunicación entre los distintos actores que intervienen, mediante 
sus modalidades sincrónica o asincrónica en el desarrollo de actividades colaborativas y en el enriquecimiento del trabajo individual y grupal para la producción del conocimiento de forma constructiva.

En este sentido, Martínez (2013) desarrolla una tipología de aprendizaje a partir de las formas interactivas con que se presentan las diferentes plataformas, de manera que para el desarrollo del aprendizaje distribuido, las aulas virtuales como Moodle tienden a ser ideales, porque favorecen la adquisición y transferencia y permiten al tutor, a los participantes y a los contenidos interactuar de manera que el aprendizaje y la enseñanza ocurran en diferentes lugares y momentos, su combinación con los sistemas semipresenciales potencian los procesos para aprender (Navarro, 2016); en el caso del aprendizaje colaborativo, los diseños bajo la noción de las herramientas web 2.0 como Facebook, Youtube, o los blogs, facilitan la interacción y distribución de la información, sabiendo que las plataformas de aulas virtuales también aportan al logro del aprendizaje de este tipo, y para el aprendizaje inmersivo, los entornos tridimensionales (Web 3D), la realidad virtual, los sistemas de simulación y modelación, los juegos educativos digitales inmersivos, en su conjunto "resultan especialmente efectivos para la educación a distancia, ya que intensifican el sentido de inmediatez y presencia social experimentado por los usuarios en un entorno en línea" (Toca, \& Carrillo, 2019, p. 16).

Este conjunto de oportunidades, ofrecido tanto por las TIC como por los EVA, ha provocado un acelerado proceso de transformación en la vida cotidiana de las personas en todo lo que va del siglo XXI (Busto, \& Coll, 2010).

A estas alturas, sería difícil negar que las Tecnologías de la Información y la Comunicación (TIC) han cambiado nuestras vidas. Sobran ejemplos que lo demuestran. Basta con pensar en la cantidad de acciones que día tras día acometemos con y a través de ellas, o en la multitud de dispositivos y terminales que se fundamentan en ellas, y de los que ya no podríamos prescindir, para pensar que, efectivamente, estamos en un nuevo lugar y en un insólito momento.

(Fuentes, Esteban, \& Caro, 2015, p. 11)

Esto ha ocasionado conflictos internos en la persona en cuanto a los nuevos esquemas que supone el desarrollo de la comprensión ante las tecnologías y las nuevas propuestas que se hacen en ella. Observándose, por ejemplo, los efectos tecnológicos en los circuitos cerebrales (Fuentes, Esteban, \& Caro, 2015), o en los cambios de hábitos en los jóvenes (Asensio, 2011), evidenciándose la infinitud de narrativas en que la persona se sumerge en su contacto con internet. Hoy día se está más cerca de comprender internet como un tejido de inteligencia compartida, donde los significados y constructos intelectuales que de ella surgen son posibles debido al sistema colaborativo desarrollado por sus usuarios. De manera, que los procesos educativos se han visto afectados por la virtualidad, sobre todo los sistemas de evaluación, la comunicación entre los estudiantes y los tutores, la planificación de las actividades y el desarrollo estratégico, y en algunos casos el abandono de los procesos por parte de los participantes o el estudiantado (Atehortúa, \& Adriana, 2010).

En los últimos años, los EVA se han perfeccionado, convirtiéndose en aplicaciones informáticas que facilitan "la comunicación pedagógica entre los participantes en un proceso educativo, sea éste completamente a distancia, presencial, o de una naturaleza mixta que combine ambas modalidades en diversas proporciones" (Adell, Castellet, \& Pascual, 2004, p. 5). A través de ellos se distribuyen materiales educativos en diferentes tipos de formatos digitales: textos, imágenes, audios, simulaciones, juegos, objetos de aprendizaje, entre otros, y su acceso permite los debates y discusiones en línea en torno a temas específicos ya sea mediante programas de cursos, asignaturas, seminarios, MOOC (Massive Online Open Courses); de igual modo, funciona para integrar contenidos alojados en las redes y facilitar la participación de expertos a través de charlas y webinars.

Las aulas virtuales, con sus dos elementos claves: la interactividad y la comunicación, facilitan la enseñanza y el aprendizaje, y profundizan en los procesos educativos desde la virtualidad mediante las modalidades de b-learning (aprendizaje semipresencial), donde se combina la entrega de los contenidos en línea con un número de sesiones presenciales; e-learning (aprendizaje en línea), donde los contenidos son entregados mediante un entorno asincrónico haciéndose uso de un software de aprendizaje a través de internet, no ocurren en ella sesiones presenciales (Bachelor, 2019), y m-learning, el cual ofrece la oportunidad de aprender mediante el uso de los dispositivos móviles (Castaño, \& Cabero, 2013).

La interactividad permite el logro de los objetivos de la enseñanza; las estrategias y métodos para este fin responden en función de procesos interactivos dados entre el estudiantado-tutor, el estudiantado-contenidos y entre los mismos miembros del estudiantado, logros que están sujetos a la capacidad de la persona para compartir y colaborar libremente con sus pares, desarrollando sus competencias mediante el uso de las herramientas tecnológicas (Martínez, López, Rodríguez, \& Martínez, 2015).

Hay que destacar que las interacciones dadas mediante las aulas virtuales generan una mayor responsabilidad por parte del estudiantado en su proceso de aprendizaje (Vázquez, Reyna, González, Hernández, \& Uvalle, 2019), siendo útil el estímulo y desarrollo de las habilidades cognitivas y metacognitivas, a fin de favorecer procesos internos en cuanto a la recuperación y facilitación de respuestas, así como el manejo de destrezas en el análisis y resolución de problemas.

En este sentido, la metacognición permite el desarrollo de habilidades durante las interacciones mediante la virtualidad, por ser una actividad mental que forma parte de las estrategias de aprendizaje y supone la capacidad de la persona para reconocer el grado de conciencia que tiene de su forma de pensar y de los procesos y eventos cognoscitivos, así como de los contenidos que subyacen en ellos (Chadwick, 1991; López, 2008; Zurita, 2020). En el contexto del desarrollo y logro de las competencia desde la virtualidad, puede llegar a facilitar la regulación, planificación, supervisión y evaluación de todas aquellas acciones implicadas en el proceso de aprendizaje (Meza, 2013). Esto hace suponer que las aulas virtuales son un entorno ideal para los procesos de formación en línea, porque permiten la interrelación entre el docente, participante, contenidos y recursos (Gros, 2011), convirtiendo su campus virtual es un entorno amigable y de fácil comprensión para el estudiantado. 


\section{Las narrativas transmedia}

La historia de la humanidad se ha sostenido en narrativas, creadas por el simple hecho de vivir en sociedades que, racionalmente, poseen un lenguaje significativo y una capacidad del habla, permitiéndole su organización social y humana (Pérez de Laborda, 2018). Siempre han existido los relatos y oportunidades para contar historias, revelándose en ellos conocimientos y estructuras cognitivas que dicen mucho de la vida de las personas: "Cada narrativa recoge críticamente circunstancias biográficas, metas, vínculos interhumanos y valoraciones profundas que constituyen el material con el que forjamos nuestra identidad en el transcurso de la vida" (Gamio, 2018, p. 295).

Las narrativas son artefactos mentales con gran impacto social, elaboradas bajo la noción de la novedad y transitoriedad, en el marco de una personalización de lo narrado (Savater, 2009). Este pensamiento narrativo, construido desde un mundo basado en significados (Bruner, 1988), permite a los seres humanos darle sentido al mundo, convirtiendo lo narrado en el mediador entre el pensamiento y la realidad cultural. El pensamiento narrativo (Adúriz, \& Revel, 2016) es el medio utilizado por las personas para construir su realidad, dándole significados a los hechos mediante dos aspectos: la secuencia de acontecimientos y la valoración de lo relatado. En tal sentido, la realidad es entendida como una secuencia de acontecimientos en el marco de un discurso donde el proceso de intercambio genera sentido y eficacia a lo narrado, permitiendo la clarificación de las visiones.

Siempre los seres humanos han hecho uso de su elocuencia para narrar historias, ya sea a través del canto, la poesía o mediante la expresión oral y corporal que supuso, por ejemplo, el teatro. Siendo ejemplo de ello las representaciones teatrales griegas:

Las representaciones teatrales griegas combinaban un buen guion, con una puesta en escena deslumbrante y unos efectos de sonido acordes a todo ello. Cada uno de los tres canales contaban la historia de diferente manera con sus propios medios coordinados armónicamente con el resto, propiciando una experiencia transmediática.

(Fuentes, Esteban, \& Caro, 2015, p. 108)

Esta descripción orienta la definición de narrativa transmedia (transmedia storytelling), término que nace en el contexto comercial y es incluido en el paradigma de la digitalización para hacer alusión a un proceso de transformación de los elementos de la ficción, los cuales son dispersados o sistematizados a través de diversos canales a fin de crear, de forma concatenada, una experiencia de entretenimiento. La idea es que cada medio utilizado contribuya significativamente a narrar la historia descrita (Jenkins, 2010), facilitando la modificación de hábitos, actitudes y conductas de la persona (Fuentes, Esteban, \& Caro, 2015).

Hoy día, las narrativas transmedia van más allá del entretenimiento, convirtiéndose no sólo en la conexión de hechos en una secuencia de causa y efecto o en el inicio y el final en una cadena de acontecimientos, sino también en una lógica discursiva concatenada mediante una serie de recursos y plataformas, así como de la intervención de los usuarios que necesitan de ello para sus fines, a objeto de generar orden a los contenidos de un proceso de formación en línea (Costa, \& Piñero, 2012).
Esta producción textual, también conocida como contenidos generados por usuarios (user-generated contents), es uno de los fenómenos más relevantes y destacados que emerge de la nueva ecología mediática. Si bien desde tiempos inmemoriales existe este tipo de práctica textual, la llegada de la web y las redes sociales ha puesto en evidencia una verdadera explosión de relatos a cargo de los prosumidores. Volviendo al concepto en cuestión, en pocos años la producción de obras transmedia, que tuvo su origen en el campo de la ficción, no tardó en pasar a la no ficción - documental transmedia, periodismo transmedia, etc. o el marketing - branding transmedia - (Scolari, Lugo, \& Masanet, 2019, p. 118).

Este salto de la narrativa transmedia desde la ficción a otros campos ha permitido el desarrollo de diferentes tipos de narrativas, surgiendo así: (a) las narrativas basadas en relatos, donde se ilustran los contenidos mediante una secuencia lógicas de hechos y procesos, pudiéndose crear personajes que narran y describen los materiales a discutir; (b) las narrativas basadas en escenarios, donde los contenidos son descritos en un escenario o estaciones sostenido en desafíos, donde el alumnado deberá tomar decisiones entre varias alternativas, ofreciéndoseles todas las herramientas teóricas necesarias para la resolución de problemas - estas narrativas están repletas de discursos motivadores a fin de incentivar al participante a lograr las metas propuestas desde el inicio-; (c) las narrativas basadas en una caja de herramientas, son propuestas formativas que permiten al estudiantado seleccionar, entre una serie de temas independientes, aquellos que resulten de mayor interés para ellos y que respondan a sus necesidades de formación, los temas son presentados en diversos tipos de plataformas y formatos; y (d) las narrativas de secuencias lógicas de contenidos, que son sistemas teóricos basados en la discusión de temas debidamente relacionados que responden a contenidos específicos y precisos aportados por un tutor o diseñador instruccional, el cual orienta el proceso y establece pautas para que los participantes hagan sus aportes haciendo uso de cualquiera de los medios disponibles en las aulas virtuales (FAO, 2014).

Mediante las narrativas transmedia los expertos orientan sus contenidos en los EVA. Estos contenidos responden a los hechos, procedimientos, conceptos, principios, actitudes y habilidades interpersonales (FAO, 2014), los mismos son narrados mediante la creativa integración de las rutas de aprendizaje, los métodos, las estrategias, los recursos y formatos, las herramientas tecnológicas y las plataformas, todo un conjunto que tiene como objetivo generar una experiencia de formación.

Cabe señalar que en la construcción de las narrativas transmedia todos los involucrados comparten la información y la reconstruyen de forma colaborativa. Cada medio o recurso hace un aporte a la construcción del mundo narrativo; evidentemente, estos aportes difieren entre sí (Scolari, 2013), admitiéndose que las nuevas narrativas surgidas en este escenario interactivo, más allá de la calidad de las mismas, son el producto de la interpretación y comprensión del conjunto.

Mediante las narrativas, las estrategias de aprendizaje son conducidas no solo por el lenguaje, si no por las plataformas y formatos utilizados, asegurándose dos situaciones: la primera, que el mensaje llegue sin interferen- 
cia, más bien de forma diáfana, clara y sencilla, y permita el desarrollo de actividades comprensivas en la medida que la persona interactúa con los contenidos y con los recursos, y, la segunda, que todas las plataformas o medios utilizados para la comprensión y el aprendizaje de los contenidos estén articuladas para tal fin, permitiendo experiencias con un alto potencial pedagógico donde se integren escenarios y medios digitales con el proceso de formación.

En educación, se ha hecho uso de las narrativas transmedia en múltiples formas, por ejemplo, como recurso digital para el aprendizaje de distintas materias y áreas educativas mediante la creación de personajes prototipos que orientan los contenidos y las estrategias de forma colaborativa, de igual modo se han utilizado juegos conducidos a través de historias que describen procesos que buscan que el estudiantado sistematice y comprenda asuntos referidos a los valores y a la ética (Villegas, 2013; Alonso, \& Murgia, 2018). De la misma manera, se han utilizado las narrativas transmedia para orientar cursos de enseñanza en diferentes áreas del conocimiento, experimentándose procesos donde el alumnado es capaz de demostrar sus habilidades cognitivas y emocionales a través de dinámicas elaboradas mediante distintos recursos y plataformas (Scolari, Lugo, \& Masanet, 2019).

La creación de narrativas transmedia supone la interrelación de las dimensiones culturales, emocionales y cognitivas, tanto de quien narra como de aquellos que con su participación co-crean los textos y discursos, entendiendo este proceso como un acto de creatividad colectiva, o sea, un sistema donde todos los involucrados favorecen, no solo la construcción de contenidos, sino también el desarrollo de las habilidades cognitivas.

En este proceso se genera un aprendizaje mutuo y cooperativo ya que entre todos se complementan los conocimientos, las habilidades y las capacidades. Al crear o compartir narraciones en un proceso co-creativo, todos los participantes aportan sus significados, intencionalidades, formas de pensar y entender la realidad, emociones, valores, propias estrategias de contar - personales o culturales - y sus conceptos o creencias del hecho educativo. La narración resultante es producto de todas estas intervenciones y de decisiones compartidas (Chomski, 2014, p. 109).

Por ello, cobra sentido la idea del aprendizaje cooperativo y colaborativo en los EVA.

El trabajo en equipo en la modalidad virtual tiene el mismo objetivo que en la modalidad presencial, es decir, el trabajo en conjunto entre un grupo de personas con intereses y objetivos comunes para maximizar no solamente su aprendizaje sino también el de los demás y favorecer la construcción colectiva de nuevos significados o enriquecer con lo que ya se cuenta.

(Martínez, 2015, p. 24)

De manera que el uso de las narrativas transmedia en las aulas virtuales responde a la necesidad de que los participantes aprendan, construyan conocimientos, interactúen y compartan experiencias. Su elaboración mediante rutas de aprendizaje, abordadas a través de diferentes métodos, ya sean estos de índole expositiva, aplicativa o colaborativa (FAO, 2014), facilita el uso y desarrollo de habilidades cognitivas y emocionales.

\section{La construcción de narrativas transmedia mediante rutas de aprendizaje}

Para Aranguren (2020) las rutas de aprendizaje son “caminos, métodos, fórmulas que elaboran el y la docente para abordar, de manera inteligente, la enseñanza en el aula. Permiten que los estudiantes pasen de un nivel cognitivo a otro y los ayuda a crear hábitos mentales para aprender" (p. 6), facilitando los procesos de adquisición, transferencia, procesamiento y sistematización de la información a fin de ayudar en la comprensión de los materiales trabajados.

El concepto de rutas de aprendizaje se ha utilizado para la creación de patrones tecnopedagógicos en la construcción de objetos de aprendizaje (Hernández-Bieliukas, \& Aranguren, 2016; Hernández-Bieliukas, Mogollón, \& Aranguren, 2019), en la producción de cursos en línea e e-learning (Alvarado, 2003), en la conexión entre los contextos estudiantiles y el currículo (Garduño, 2020), en el diseño de proyectos colectivos de índole socioeconómica y cultural (Muñoz, 2008), y como lineamientos para orientar la acción "pedagógica y didáctica para una enseñanza efectiva de las competencias curriculares" (Ministerio de Educación de Perú, 2015, p. 5). Por ello, su acertada concepción y utilización en los procesos de enseñanza permite el procesamiento y transferencia de la información de forma adecuada, sistematizándola como un conocimiento.

Existen diversos medios para la presentación de una ruta de aprendizaje, uno de ellos es mediante las infografías que permiten la visualización gráfica de un sistema conducente al logro de objetivos en el desarrollo de metas en cuanto al aprendizaje se refiere, mecanismo interrelacionado con los llamados mapas de progresos (Latorre, 2014). Otra forma está basada en la concepción integrada de los contenidos, estrategias y métodos presentados en las narrativas de los cursos diseñados en las diferentes plataformas de e-learning (Alvarado, 2003).

Estos mecanismos de presentación y concepción de las rutas de aprendizaje conducen a pensar en la necesidad de medios que faciliten el logro y la evaluación de las competencias en el marco del desarrollo de habilidades cognitivas y de comprensión. El uso de los enfoques cognitivos en la construcción de rutas de aprendizaje y, sobre todo, en aquellas orientadas a la elaboración de narrativas transmedia, son fundamentales para ayudar a la persona que aprende a guiar su proceso autodidacta.

Todas estas propuestas poseen nociones diferentes acerca de lo que se supone es una ruta de aprendizaje; de todas ellas hay que destacar la concepción abordada por Hernández-Bieliukas, Mogollón y Aranguren (2019), que la consideran como el diseño cognitivo que responde a teorías específicas y que orientan a un flujo concatenado de actividades. Es bajo este precepto que se propone la construcción de narrativas transmedia utilizadas para el desarrollo de los procesos de aprendizaje en las aulas virtuales.

En este contexto del análisis, la construcción de narrativas transmedia requiere del aporte de cada medio o plataforma que sobre ellas actúa (Scolari, 2013). Las aulas virtuales están diseñadas bajo la orientación del aprendizaje autodirigido, entendiendo esto como el proceso de aprendizaje conducido por la misma persona que incluye la "autoconciencia (metacognición), y capacidades para 
la autorregulación del proceso de aprendizaje y su autoevaluación, las cuales pueden establecer las bases para el autocontrol" (Chadwick, 1988, p. 167). En tal sentido, las narrativas deben ser conducidas mediante una secuencia didáctica basada en Actividades de Apertura (AA), Actividades de Desarrollo (AD) y Actividades de Cierre (AC) (Díaz Barriga, 2013), y elaboradas a partir de las competencias, objetivos y estrategias de desarrollo de habilidades cognitivas de procesamiento y ejecución (Gilar, 2003).

Tanto la construcción de las narrativas como de las rutas de aprendizaje, tienen como punto de partida los planes de estudio o programas de cada una de las instituciones, por lo que deben responder ya sea a la asignatura, módulo, unidad de aprendizaje o cualquier otra denominación curricular establecida para definir los contenidos. Es sobre la secuencia didáctica, que en sí misma representa una ruta, donde se orienta la ruta de aprendizaje basada en las estrategias de procesamiento y ejecución, teniendo como resultado la adquisición de habilidades cognitivas y la comprensión del material estudiado.

Las estrategias de procesamiento incluyen las actividades que la persona que aprende debe hacer para atender e ingresar de manera exitosa la información a su memoria, y las de ejecución representan aquellas que permiten la recuperación de la información, la formulación de respuestas, la generalización y la resolución de problemas, "la diferencia entre los dos grupos puede ser vista como estrategias de entrada vs estrategias de salida" (Chadwick, 1988, p. 167).

En tal sentido, las narrativas que conducen los proceso y sistemas de formación y aprendizaje en las aulas virtuales, deben orientar al participante a que realice tareas y actividades de índole cognitiva tales como la reflexión, diferencias, semejanzas, inferencias, contrastaciones, parafraseo, creación de imágenes mentales, construcción de redes de aprendizaje, resolución de problemas, desarrollo y aplicación de la creatividad, sistematización de procesos, experiencias y sistemas, construcción de hipótesis y teorías y evaluación de procesos-sistemas (Chadwick, 1991; Gilar, 2003), así como demostrar el alcance de la comprensión de los contenidos trabajados a través de la explicación, la ejemplificación, la relación, la aplicación, la comparación, el contraste, la justificación, la contextualización y la generalización (Perkins, 1999). Esto se logra mediante un diseño instruccional debidamente planificado (Alvarado, 2003), donde las narrativas estén construidas considerando, para cada una de ellas, una ruta de aprendizaje basada en actividades cognitivas y de comprensión que responden a las necesidades reales de aprendizaje, formación y capacitación de la persona.

De manera que la ruta de aprendizaje se distribuirá en toda la secuencia didáctica, por lo que cada estrategia o actividad seleccionada será parte de la narrativa transmedia. Cada actividad de desarrollo de habilidades, tanto de procesamiento como de ejecución debe ser conducida por recursos, objetos de aprendizaje, herramientas multimedia o tecnológicas y por la participación entre pares, por lo que la metodología que se desarrollará debe estar dirigida por procesos de índole colaborativo y cooperativo (Martínez, 2015).

Para visualizar este modelo de construcción de narrativas transmedia basada en rutas de aprendizaje, obsérvese la Figura 1.

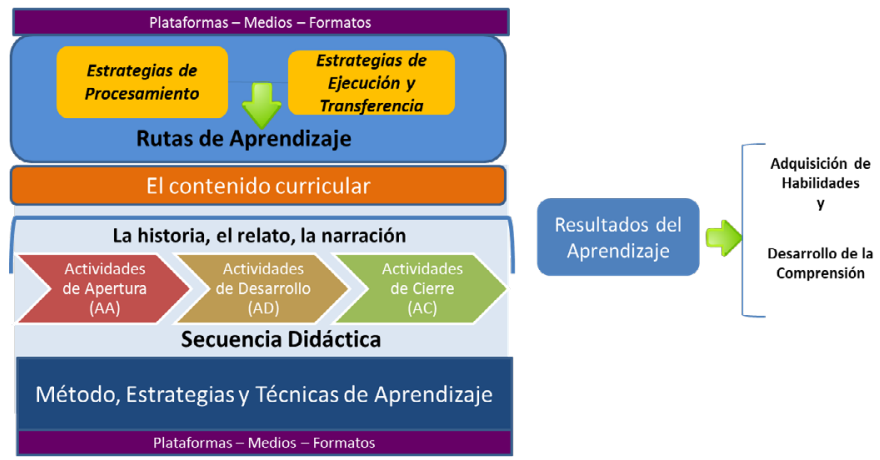

Fig. 1. Modelo para la construcción de narrativas transmedia basado en rutas de aprendizaje para aulas virtuales.

\section{Implementación y Aplicabilidad}

Para Ríos (2016), los procesos de formación en la educación virtual representan en sí mismos una ruta de aprendizaje "a través del cual los alumnos y profesores interactúan basados en el modelo cooperativo que promueve la intervención de ambos actores durante el proceso" ( $p$. 115), revelándose en ellos las narrativas transmedia como una herramienta para facilitar el desarrollo de los contenidos mediante diferentes y múltiples canales y plataformas como páginas web, televisión, cómic, videojuegos, entre otros. En tal sentido, para la construcción e implementación de las narrativas transmedia en las aulas virtuales es necesario destacar tres componentes esenciales: la narrativa (storytelling), la audiencia y la selección de medios o plataformas, y formatos.

\subsection{La narrativa (storytelling).}

Existen diversas propuestas acerca del uso de las narrativas para la enseñanza, ya sea de la lengua (Aparicio, \& Pérez, 2020), como de las ciencias (Adúriz, \& Revel, 2016), en tal sentido el uso del storytelling como técnica para el desarrollo de relatos requiere de un estricto diseño y planificación de manera que la historia construida impacte y permita, en el participante, motivación e interés en la continuidad del aprendizaje, autonomía, desarrollo de competencias y de una experiencia emocional facilitadora de la imaginación y la creatividad, permitiendo que la persona se involucre ya sea con los personajes o con las secuencias narrativas, de modo que las ilustraciones, discursos y recursos contribuyan a la adquisición de habilidades cognitivas y de comprensión, garantizándose el aprendizaje colaborativo a partir de las interacciones (Aparicio, \& Pérez, 2020).

Las narrativas están diseñadas con tres componentes: el contexto, la historia, y los personajes y relaciones.

El contexto de la narrativa es el espacio, tanto geográfico como temporal, donde se desarrolla el relato. En él se describen los acontecimientos, hechos o fenómenos que estudiar, que no son más que la historia que puede ser contada ya sea de forma lineal o ramificada (Rosendo, 2019), o a través de una narración que hace uso del tiempo mediante saltos, ya sea del pasado al futuro o del presente al pasado, dependiendo de los casos y de las necesidades. En las historias encontramos, de forma implícita o explícita, los contenidos que desarrollar. En los personajes y relaciones descansan, básicamente, todas las acciones que se describen en la historia. 
Un aspecto importante en la construcción de la narrativa es la planificación y organización del proceso, para ello se requiere considerar los siguientes elementos:

a) Las competencias, las cuales deberán estar definidas a partir de las exigencias curriculares;

b) La presentación de la narrativa, ya sea en forma de relato, basada en escenarios, en forma de caja de herramientas o en secuencias lógicas de contenidos;

c) Los contenidos, que orientan las historias deben presentarse mediante una secuencia didáctica, que dirija no solo la historia misma, sino las actividades y tareas, mediante sus tres momentos puntuales: inicio, desarrollo y cierre;

d) La ruta de aprendizaje, la cual se define a partir de las actividades cognitivas y de comprensión que se requieren para alcanzar las competencias establecidas;

e) Las actividades y tareas que los participantes han de desarrollar, las cuales deben construirse tomando en cuenta los métodos de enseñanza virtual: expositivo, aplicativo y colaborativo, de manera que se evidencie, a través de ellos, los aportes de cada uno de los participantes y las aplicaciones de las actividades cognitivas propuestas en la ruta de aprendizaje;

f) Los medios o plataformas, y formatos a utilizar.

Todos estos elementos deben estar integrados de manera creativa con el objeto de facilitar el aprendizaje.

\subsection{Definición de la audiencia}

Es menester identificar la audiencia hacia la cual se va a orientar la narrativa. Siendo importante precisar perfiles socioeconómicos, tecnológicos y afectivos, que orienten la acción pedagógica que se ha de planificar y organizar en virtud de las narrativas. Hay que destacar cómo la audiencia contribuiría al desarrollo de los contenidos.

\subsection{Selección de los medios/plataformas y de los formatos nece-} sarios para la construcción de las narrativas transmedia

Para Scolari (2013) en el mundo de la narrativa transmedia las historias deben fluir de un medio a otro, por lo que es necesario valorar el uso de los diferentes medios para que las historias sean contadas de diversos modos, generando experiencias satisfactorias en el aprendizaje y en el desarrollo cognitivo de la persona. Es imprescindible el aprovechamiento de las características específicas de cada medio, por ejemplo, el uso del potencial ofrecido por el cine para la enseñanza en asuntos referidos a la ciencia (Aranguren, 2018).

Entre los medios o plataformas que se pueden utilizar para la construcción de narrativas transmedia en las aulas virtuales se encuentran las siguientes: la televisión con su diversidad de programación, así como la variedad de canales ofrecidos por Youtube, con innumerables programaciones; el cine, pudiéndose utilizar el formato de video para la presentación de este tipo de materiales; el uso de libros y la construcción de textos escritos, o de audios (podcast) con discursos o narraciones que faciliten la comprensión de los contenidos; la creación de foros y la diversidad de presentaciones de las distintas opiniones de los que participan ayudan mucho a entender cómo sistematizan los participantes las informaciones; la utilización de sitio web específicos y de redes sociales (WhatsApp, Instagram Youtube, Telegram, entre otros) permiten la difusión de los contenidos y facilita la comprensión de los mismos, todo esto integrado en un discurso narrado, debidamente planificado y organizado, en el aula virtual mediante métodos de enseñanza, tareas, actividades y rutas que orienten el aprendizaje y la consolidación de habilidades y destrezas.

\section{Conclusiones}

Las aulas virtuales se han convertido en una oportunidad para la formación, capacitación y enseñanza elearning. En ellas los contenidos, objetivos, actividades y prácticas, así como las evaluaciones están concatenados de manera que el estudiantado puede acceder con facilidad. Estos EVA representan una herramienta tecnológica didáctica que facilita las interacciones entre los participantes y sus tutores y con las plataformas, con el fin de que la persona construya conocimientos y aprenda.

En las aulas virtuales se desarrollan dos elementos fundamentales que son la interactividad y la comunicación. Bajo esta premisa se concretan procesos educativos virtuales mediante diferentes modalidades como el e-learning, representado por el aprendizaje en línea y de forma asincrónica, el b-learning que representa el aprendizaje semipresencial y el m-learning, sostenido mediante el uso de dispositivos móviles, todo ello tiene como fin la profundización de los procesos de formación y capacitación profesional, por lo que el diseño de los procesos formativos deben estar orientados por narrativas que satisfagan el aprendizaje de la persona.

Las narrativas transmedia están representadas por aquellas historias que surgen mediante múltiples y diferentes plataformas. Son formas de narrar que pueden expandirse mediante diferentes sistemas de significación, verbal, icónico, audiovisual, interactivo, entre otros, y medios como el cine, la televisión, video juegos, podcast, etc., favoreciendo las interacciones y la co-creación mediante el desarrollo del aprendizaje colaborativo. El uso de rutas de aprendizaje en su construcción facilita la orientación del proceso y el logro preciso de las competencias y objetivos.

La idea de construir narrativas en las aulas virtuales responde a la necesidad de sostener los discursos para la enseñanza virtual bajo una lógica transmedia, basada en la capacidad de conexión con otros sistemas que puedan nutrirlos y hacer de estos procesos algo más efectivo y cónsono con las nuevas realidades sociales y educativas. De manera, que las rutas de aprendizaje deben convertirse en herramientas cognitivas que faciliten la conexión de diferentes medios y formatos para conducir la enseñanza y facilitar el aprendizaje.

Con las rutas de aprendizaje se asegura el logro de las competencias y se brindan herramientas para que la persona que aprende las valore como modelos metacognitivos. Ser consciente de lo que se hace facilita el éxito en el logro del aprendizaje; por lo tanto, la metacognición, que supone la regulación de la cognición y supervisión del aprendizaje mediante la planificación, monitoreo de las actividades de aprendizaje y la evaluación de la eficiencia y eficacia, tanto de las acciones como de los resultados (Valenzuela, 2019), es de suma importancia en el desarrollo cognitivo de aquellas personas que experimentan procesos de autogestión y formación a través de las aulas virtuales. 
Las narrativas transmedia requieren de estrategias colaborativas en línea. Esto supone una reorganización de los procesos de aprendizaje. La naturaleza de la educación virtual plantea que los seres humanos son capaces de aprender desde distintos lugares y tiempos diferentes, a través de intercambio e interacción social para despertar procesos internos que sean transferidos a contextos reales. No puede dejarse a un lado el uso de actividades cognitivas en la construcción de narrativas, porque aportan sentido a los procesos o sistemas de formación, capacitación y aprendizaje que se pretenden trabajar en aulas virtuales, $\mathrm{o}$ en cualquier otro entorno de aprendizaje.

Las narrativas transmedia orientan la realización de tareas y actividades de índole cognitiva, permitiéndole al participante de la formación en línea, la demostración de su capacidad comprensiva ante el desarrollo de cualquier contenido a través de actividades individuales o colectivas.

Si se requiere construir una narrativa transmedia que aborde cualquier contenido para un curso en una plataforma de e-learning, se deberá precisar, a partir de las competencias $\mathrm{u}$ objetivos definidos, una lista secuencial de las actividades de procesamiento y ejecución que se desarrollarán; esta secuencia de actividades representaría la ruta de aprendizaje que estará distribuida a lo largo de toda la secuencia didáctica; se deberán establecer y describir los métodos que se utilizarán para el logro de cada actividad de procesamiento y ejecución, a su vez, se deben definir los medios, plataformas y formato a utilizar para desarrollar la narrativa.

En cuanto a las valoraciones de estos procesos, la construcción de los ítems de evaluación debe responder a cada una de las actividades cognitivas y de comprensión propuestas en la ruta de aprendizaje y, por supuesto, a las competencias $\mathrm{u}$ objetivos definidos al inicio del proceso.

\section{Referencias}

Adell, J., Castellet J., \& Pascual, J., (2004). Selección de un entorno virtual de enseñanza/aprendizaje de código fuente abierto para la Universitat Jaume I. Barcelona: Centre d'Educació i Noves Tecnologies (CENT) de la Universitat Jaume I.

Adúriz, A., \& Revel, A. (2016). El pensamiento narrativo en la enseñanza de las ciencias. Inter Ação, 41(3) 691704. doi:10.5216/ia.v41i3.41940

Alonso, E., \& Murgia, V. A. (2018). Enseñar y aprender con narrativas transmedia. Análisis de experiencia en una escuela secundaria de Argentina. Comunicación y Sociedad, 33, 203-222. doi:10.32870/cys.v0i33.7039

Alvarado, A. (2003). Diseño Instruccional para la producción de cursos en línea y e-learning. Docencia Universitaria, 1(4), 9-24.

Aparicio, Y., \& Pérez, M. (2020). Storytelling. La lectura de álbumes ilustrados en la enseñanza de la lengua inglesa. Madrid: Pirámide.

Aranguren, G. (2018). La estrategia verbal de enseñanza y el aprendizaje cooperativo integrado con películas de ciencia ficción. Revista de Pedagogía, 39(104), 9-29.

Aranguren, G. (2020). Lineamientos estratégicos para la consolidación de una escuela inteligente y su noción de felicidad. Revista de Educación, 44(2). doi:10.15517/ revedu.v44i2.37605
Asensio, J. M. (20-22 de octubre, 2011). Plasticidad, nuevas tecnologías y cambios mentales ¿qué pedagogía? XII Congreso Internacional de Teoría de la Educación. Barcelona, España.

Atehortúa A., \& Adriana A. (2010). Dificultades de los estudiantes en la adaptación de la metodología de educación a distancia en la Universidad Nacional Abierta y a Distancia UNAD, CERES Villa de San Sebastián de la Plata, Huila. Revista de Investigaciones UNAD, 9(2), 55-78. doi:10.22490/25391887.672

Bachelor, J. W. (2019). El aula presencial, semipresencial, virtual e invertida: Un estudio comparativo de métodos didácticos en la enseñanza de L2. Revista de Educación. 43, (2). doi:10.15517/ revedu.v43i2.34014

Bautista, A. (2004). Las nuevas tecnologías en la enseñanza. Temas para el usuario. Madrid: Akal.

Bruner, J. (1988). Realidad mental y mundos posibles. Barcelona: Gedisa.

Busto, A., \& Coll, C. (2010). Los entornos virtuales como espacio de enseñanza y aprendizaje. Una perspectiva psicoeducativa para su caracterización y análisis. RMIE, 15(44), 163-184.

Castaño, C., \& Cabero, J. (2013). Enseñar y aprender en entornos m-learning. Madrid: Síntesis.

Castejón, J. L., \& Gilar, R. (2005). Evaluación del estilo de enseñanza-aprendizaje en estudiantes universitarios. Revista de Psicología y Educación, 1(2), 137-152.

Chadwick, C. B. (1991). Una revolución verde en la educación: Las estrategias de aprendizaje. Revista de Psicología, 9(1), 3-14. doi:10.18800/psico.199101.001

Chadwick, C. B. (1988). Estrategias cognoscitivas y afectivas del aprendizaje. Revista Latinoamericana de Psicología, 20(2), 163-184.

Chomski, D. (2014). EduStorytelling. El arte de contar historias para la vida cotidiana y los aprendizajes. Barcelona: UOC.

Colás, M. P. (Noviembre de 2006). Metodología pedagógica para e-learning desde un enfoque sociocultural. En Metodología Pedagógica para e-learning, VI Conferencia Internacional sobre E-learning y Tecnologías de Educación. Lisboa: Portugal.

Costa, C., \& Piñero, T. (2012). Nuevas narrativas audiovisuales: multiplataforma, crossmedia y transmedia. El caso de Águila Roja (RTVE). ICONO 14, 1(2), 102-125. doi:10.7195/ri14.v10i2.156

Díaz Barriga, Á. (2013). Secuencias de aprendizaje. ¿Un problema del enfoque de competencias o un reencuentro con perspectivas didácticas? Profesorado. Revista de Currículum y Formación de Profesorado, 17(3), 11-33.

Domínguez, R. (2016). Los entornos personales de aprendizaje. En M. L. Cacheiro, R., Sánchez, y L. González (coords.), Recursos tecnológicos en contextos educativos (pp. 135 - 159). Madrid: Universidad Nacional de Educación a Distancia.

Fernández-Pampillón, A. (2009). Las plataformas e-learning para la enseñanza y el aprendizaje universitario en Internet. En M. C. López Alonso (ed.) y M. Matesanz del Barrio (coord.), Las plataformas de aprendizaje. Del mito a la realidad (pp. 45 - 76). Madrid: Biblioteca Nueva.

Fuentes, J. L., Esteban, F., \& Caro, C. (2015). Vivir en Internet. Retos y reflexiones para la educación. Madrid: Síntesis. 
Gamio, G. (2018). Narrativas y metánoia. El proceso de cambio de perspectiva basado en transiciones. En C. Monteagudo, y P. Quintanilla. Los caminos de la filosofía. Diálogo y Método. Lima: Pontificia Universidad Católica del Perú/Fondo Editorial.

García, L. (2018). Blended learning y la convergencia entre la educación presencial y a distancia. RIED. Revista Iberoamericana de Educación a Distancia, 21(1), 09-22. doi:10.5944/ried.21.1.19683

García-Valcárcel, A., Muñoz-Repiso. A., \& Hernández, A. (2013). Recursos tecnológicos para la enseñanza e innovación educativa. Madrid: Síntesis.

Garduño, E. (2020). Rutas de aprendizaje en la inducción, ingreso y seguimiento de un proceso de formación. Revista Educación, 44(2). doi:10.15517/revedu.v44i2.38859

Gil, J., \& Mataveli, M. (2018). Medios y redes para el aprendizaje. En M. L. Cacheiro, Educación y Tecnología: Estrategias didácticas para la integración de la TIC (pp. 161-181). Madrid: Universidad Nacional de Educación a Distancia.

Gilar, C. (2003). Adquisición de habilidades cognoscitivas. Factores en el desarrollo inicial de la competencia experta. [Tesis Doctoral]. Universidad de Alicante.

Gros, B. (2011). El modelo educativo basado en la actividad de aprendizaje. En B. Gros (ed.), Evolución y retos de la educación virtual construyendo el e-learning del siglo XXI (pp. 13 - 26). Barcelona: UOC.

Hernández-Bieliukas, Y., \& Aranguren, G. (2016). Patrón tecnopedagógico: ruta de aprendizaje basado en actividades comprensivas. Revista Vínculos, 13(2), 30-39.

Hernández-Bieliukas, Y., Mogollón, I., \& Aranguren, G. (2019). Patrón tecnopedagógico: ruta de aprendizaje para el desarrollo de habilidades cognitivas. En T. E Vaquero, B. E. Brescó, R. J. I. Coiduras, y F. F.X. Carrera (comps.), Educación con tecnología. Un compromiso social. Iniciativas y resultados de investigaciones y experiencias de innovación educativa (pp. 2161 - 2177). Lleida: Universitat de Lleida. doi:10.21001/edutec. 2019

Jenkins, H. (2010). Transmedia Storytelling and Entertainment: An annotated syllabus. Continuum, 24(6), 943958. doi:10.1080/10304312.2010.510599

Latorre, M. (2014). Mapas de progresos del aprendizaje (MPA) y Rutas de Aprendizaje (RA) en Perú - 2013. Revista de Investigación en Psicología, 16(1), 211-231. doi:10.15381/rinvp.v16i1.3928

López, O. (2008). La Inteligencia emocional y las estrategias de aprendizaje como predictores del rendimiento académico en estudiantes universitarios [Tesis de Maestría]. Universidad Nacional Mayor de San Marcos. Lima, Perú.

Malbrán, M. C. (2012). La educación a distancia(EAD): Una perspectiva cognitiva. Signos Universitarios, 31(48), 43-52.

Martínez, N. L. (2015). El trabajo en equipo como estrategia de aprendizaje en ambientes virtuales. En L. Galindo, El aprendizaje colaborativo en ambientes virtuales (pp. 15 - 39). Guadalajara: CENID.

Martínez, R. (2013). Sloodle. Conexión de entornos de aprendizaje. Barcelona: UOC.

Martínez, J., López, G., Rodríguez, V., \& Martínez, A. L. (2015). El Aprendizaje Distribuido. Primer paso a la virtualidad en la Enseñanza en el Nivel Universitario.
PAG, Revista Iberoamericana de Producción Académica y Gestión Educativa, 2(3), 1-14.

Meza, A. (2013). Estrategias de aprendizaje. Definiciones, clasificaciones e instrumentos de medición. Propósitos y Representaciones, 1(2), 193-213. doi:10.20511/pyr2013. v1n2.48

Ministerio de Educación de Perú. (2015). Rutas de Aprendizaje. Versión 2015. ¿Qué y cómo aprenden nuestros niños y niñas? II Ciclo. Área curricular: Comunicación. Lima: Ministerio de Educación.

Monroy, A.; Hernández, I. A. y Jiménez, M. (2018). Aulas Digitales en la Educación Superior: Caso México. Revista Formación Universitaria, 11(5), 93-104. doi:10.4067/ S0718-50062018000500093

Moreno, J. (2018). Las TIC en el proceso de enseñanza y aprendizaje. En M. L. Cacheiro, Educación y Tecnología: Estrategias didácticas para la integración de las TIC (pp. 6-26). Madrid: Universidad Nacional de Educación a Distancia.

Morín, E. (1999). Los siete saberes necesarios para la educación del futuro. París: UNESCO.

Muñoz, J. P. (2008). Las rutas de aprendizaje: una estrategia novedosa de desarrollo rural con identidad territorial. El caso de Cauca-Nariño (Colombia). Cuadernos de Desarrollo Rural, 5(60), 113-132.

Navarro, M. (2016). Aprendizaje distribuido en red y diseño instruccional en programas de postgrados [Documento en línea].

Olarte, S. (2017). Brecha digital, pobreza y exclusión social. Temas Laborales, 138, 285-313.

Organización de las Naciones Unidas para la Alimentación y la Agricultura (2014). Metodologías de E-learning. Una guía para el diseño y desarrollo de cursos de aprendizaje empleando tecnologías de la información y las comunicaciones. Roma: FAO.

Organización Panamericana de la Salud. (2020, marzo 16). Orientación ética sobre cuestiones planteadas por la pandemia del nuevo coronavirus (COVID-19).

Ortiz, E. F. (2018). Narrativas transmedia en el aula de clase: reconocimiento, disputa y construcción permanente. Educación y Ciudad, 35, 179-188. doi:10.36737/01230425. V0.N35.2018.1973

Pérez de Laborda, M. (2018). ¿Qué es el hombre? En M. Pérez De Laborda, F. J. Soler, y C. E. Vanney (comps.) (pp. 4-7). ¿Quiénes somos? Cuestiones en torno al ser humano. Pamplona: Ediciones de la Universidad de Navarra.

Perkins, D. (1999). La escuela inteligente. Barcelona: Gedisa.

Ralón, L., Vieta, M., \& Vásquez, M. L. (2004). De(formación en línea): acerca de las desventajas de la educación virtual. Comunicar, 22, 171-176. doi:10.3916/C22-200426

Reyero, M. (2019). La educación constructivista en la era digital. Revista Tecnología, Ciencia y Educación, 12, 111127.

Ríos, I. N. (2016). Uso de la narrativa transmedia en entornos académicos virtuales o bimodales. Miradas, 1(14), 113-121. doi:10.22517/25393812.15641

Rosendo, N. (2019). Proceso de ideación de contenidos didácticos empleando el transmedia storytelling. Puntos de entrada, narrativa, experiencia, plataformas, canales, medios. Storytelling y transmedia en e-learning. Proyecto Open Course Ware-UNIA, Universidad Internacional de Andalucía. 
Santoveña-Casal, S. (2019). Análisis de pedagogía digitales. Comunicación, Redes Sociales y nuevas narrativas. Barcelona: Octaedro.

Savater, F. (2009). La tarea del héroe. Barcelona: Ariel.

Scolari, C. A. (2013). Narrativas transmedia. Cuando todos los medios cuentan. Barcelona: Centro Libros PAPF.

Scolari, C A., Lugo, N, \& Masanet, M J. (2019). Educación Transmedia. De los contenidos generados por los usuarios a los contenidos generados por los estudiantes. Revista Latina de Comunicación Social, 74, 116-132. doi:10.4185/RLCS-2019-1324

Silva, J. (2011). Diseño y moderación de entornos virtuales de aprendizaje. Barcelona: UOC, Editorial.

Tapia, C. E. (2016). El aprendizaje en la educación a distancia [documento en línea].

Toca, C. E., \& Carrillo, J. (2019). Los entornos de aprendizaje inmersivos y la enseñanza a ciber-generaciones. Educaçao e Pesquisa, 45(1), 1-20. doi:10.1590/s16784634201945187369
Valenzuela, M. A. (2019). ¿Qué hay de nuevo en la metacognición? Revisión del concepto, sus componentes y términos afines. Educaçao e Pesquisa, 45 (1), 1-20. doi: 10.1590/S1678-4634201945187571

Vázquez E., Reyna J., González N. I., Hernández, M. A., \& Uvalle, Y. (2019). Las TIC como estrategia didáctica innovadora para el diseño del curso: historia de la educación en México del primer semestre de la licenciatura en educación primaria. En: E. Vaquero, E. Brescó, J. Coiduras, y X. Carrera. Educación con tecnología. Un compromiso social. Iniciativas y resultados de investigaciones y experiencias de innovación educativa (pp. 1795-1810). Lleida: Ediciones de la Universidad de Lleida. doi:10.21001/ edutec.2019

Villegas, I. (2013). Los usos educativos de las narrativas transmedia. Barcelona: Gabinete de Comunicación y Educación.

Zurita, M. S. (2020). El aprendizaje cooperativo y el desarrollo de las habilidades cognitivas. Revista Educare, 24(1), 51-74. doi:10.46498/reduipb.v24i1.1226

Copyright: (C) 2021 Aranguren Peraza. Este es un artículo de acceso abierto distribuido bajo la licencia Creative Commons de Atribución No Comercial 4.0, que permite su uso sin restricciones, su distribución y reproducción por cualquier medio, siempre que no se haga con fines comerciales y el trabajo original sea fielmente citado. 